Article

\title{
The Segal-Bargmann Transform for Odd-Dimensional Hyperbolic Spaces
}

\author{
Brian C. Hall ${ }^{1, \dagger}, *$ and Jeffrey J. Mitchell ${ }^{2, \dagger}$ \\ ${ }^{1}$ Department of Mathematics, University of Notre Dame, 255 Hurley Hall, \\ Notre Dame, IN 46556, USA \\ ${ }^{2}$ Department of Mathematics, Robert Morris University, 6001 University Boulevard, \\ Moon Township, PA 15108, USA; E-Mail: mitchellj@ rmu.edu \\ $\dagger$ These authors contributed equally to this work. \\ * Author to whom correspondence should be addressed; E-Mail: bhall@nd.edu \\ Academic Editor: Palle E.T. Jorgensen
}

Received: 7 July 2015 / Accepted: 10 August 2015 / Published: 18 August 2015

\begin{abstract}
We develop isometry and inversion formulas for the Segal-Bargmann transform on odd-dimensional hyperbolic spaces that are as parallel as possible to the dual case of odd-dimensional spheres.
\end{abstract}

Keywords: Segal-Bargmann transform; heat kernel; hyperbolic space; spherical function

\section{Introduction}

The Segal-Bargmann transform for Euclidean space was developed in the 1960s as a unitary map from $L^{2}\left(\mathbb{R}^{n}\right)$ to an $L^{2}$ space of holomorphic functions on $\mathbb{C}^{n}$ with respect to a Gaussian measure [1-3]. Motivated by work of Gross [4], the first author introduced an analog of the Segal-Bargmann transform for compact Lie groups and proved isometry and inversion formulas for it [5-7]. The transform is connected to the Segal-Bargmann transform on an infinite-dimensional Euclidean space [8] and can be extended to the group of paths with values in a compact group [9]. The transform also arises in the study of two-dimensional Yang-Mills theory on a spacetime cylinder [10-12] and in geometric quantization [13] and has been used in the study of quantum gravity (see [14,15] among many others). 
The results of [5,6] were extended to the case of a compact symmetric space by Stenzel in [16]. Let $U$ be a simply connected compact group; let $K$ be the fixed-point subgroup of an involution; and let $X=U / K$ (every simply connected compact group can be thought of in this way; other examples include spheres and projective spaces). If $X_{\mathbb{C}}:=U_{\mathbb{C}} / K_{\mathbb{C}}$ is the "complexification" of $X$, there is a diffeomorphism $\Phi: T(X) \rightarrow X_{\mathbb{C}}$ given by:

$$
\Phi(x, Y)=\exp _{x}(i Y)
$$

where the right-hand side of the formula refers to the analytic continuation of the geometric exponential map. The image of a single fiber in $T(X)$ in $X_{\mathbb{C}}$ may be identified with the dual non-compact symmetric space to $X$. If, for example, $X$ is an $n$-sphere, the image of each fiber can be identified with $n$-dimensional hyperbolic space.

Now, for each $t>0$, we define the Segal-Bargmann transform $C_{t}: L^{2}(X) \rightarrow \mathcal{H}\left(X_{\mathbb{C}}\right)$ by:

$$
C_{t}(f)=\left(e^{t \Delta / 2} f\right)_{\mathbb{C}}
$$

where $e^{t \Delta / 2}$ is the (forward) heat operator on $X$ and $(\cdot)_{\mathbb{C}}$ denotes analytic continuation in the space variable from $X$ to $X_{\mathbb{C}}$. If $f \in L^{2}(X)$ and $F=C_{t}(f) \in \mathcal{H}\left(X_{\mathbb{C}}\right)$, the isometry and inversion formulas of [16] take the following form:

$$
\begin{aligned}
\|f\|^{2} & =\int_{x \in X} \int_{Y \in T_{x}(X)}\left|F\left(\exp _{x}(i Y / 2)\right)\right|^{2} v_{2 t}(Y) J(Y) d Y d x \\
f(x) & =\int_{Y \in T_{x}(X)} F\left(\exp _{x}(i Y)\right) v_{t}(Y) J(Y) d Y .
\end{aligned}
$$

Here, $\nu_{t}$ and $J$ are the heat kernel and the Jacobian of the exponential, respectively, for the dual non-compact symmetric space to $X$. Note that in Equation (1), we have $\exp _{x}(i Y / 2)$ and $v_{2 t}(Y)$, whereas in Equation (2), we have $\exp _{x}(i Y)$ and $v_{t}(Y)$.

It is natural to attempt to extend the isometry and inversion formulas to the case where $X$ is a non-compact symmetric space. In light of the duality between compact and non-compact symmetric spaces, one would expect, roughly speaking, to reverse the roles of compact and non-compact in Equations (1) and (2). In attempting to do this, however, substantial complications quickly arise. The polar decomposition, for example, is no longer a diffeomorphism, and functions of the form $e^{t \Delta / 2} f$ do not extend to all of $X_{\mathbb{C}}$, but only to the Akhiezer-Gindikin "crown domain" [17] (see [18,19]).

One way to work around these difficulties was developed by Krötz, Ólafsson and Stanton in [20]. Let $G$ be a connected semisimple Lie group with finite center, and let $K$ be a maximal compact subgroup of $G$, so that $G / K$ (with a $G$-invariant metric) is a Riemannian symmetric space of the non-compact type. If $f$ is in $L^{2}(G / K)$ and $F=e^{t \Delta / 2} f$, one defines the orbital integral, given by:

$$
\mathcal{O}_{|F|^{2}}(i Y)=\int_{G}\left|F\left(g \cdot \exp _{x_{0}}(i Y / 2)\right)\right|^{2} d g
$$

for $Y$ in the tangent space $\mathfrak{a}$ to a maximal flat through the base point $x_{0}$. This function is initially defined only for $Y$ in a certain bounded domain $2 \Omega$, and it blows up on the boundary of $2 \Omega$. Nevertheless, there is a pseudodifferential "shift operator" $D$ on $\mathfrak{a}$ that can be used to eliminate the singularities in $\mathcal{O}_{|F|^{2}}$. The operator $D$ is defined, essentially, by requiring that it map the spherical functions for $G / K$ into their 
Euclidean counterparts, which are just linear combinations of exponentials (compare Equation (3.11) to Equation (3.12) in [20]). We are then interested in the operator $\tilde{D}$, which describes the action of $D$ on the imaginary axis; that is, $\tilde{D}$ satisfies:

$$
(D g)(i Y)=\tilde{D}(g(i Y))
$$

It is shown in [20], using the Gutzmer-type formula of Faraut [21], that $\tilde{D} \mathcal{O}_{|F|^{2}}$ extends without singularities to all of $\mathfrak{a}$.

The isometry formula of [20] (Theorem 3.3) then reads:

$$
\|f\|^{2}=\int_{\mathfrak{a}} \tilde{D}\left[\mathcal{O}_{|F|^{2}}(i Y)\right] w_{t}(Y) d Y
$$

where:

$$
w_{t}(Y)=e^{t|\delta|^{2}} \frac{e^{-|Y|^{2} /(4 t)}}{(4 \pi t)^{k / 2}} .
$$

Here, $k=\operatorname{dim} \mathfrak{a}$ and $\delta$ is half the sum of the positive roots with multiplicity. We adjust the formula in [20] to fit our normalization of the heat equation and to correct for a minor inconsistency in [20] in how the orbital integral is defined. We also use a different normalization of the shift operator, which means that we do not need a factor of the order of the Weyl group in the definition of $w_{t}$, as in [20]. See also [22,23] for a different approach to the isometry formula on $G / K$, but which also involves a sort of shift operator.

On the one hand, the isometry formula in Equation (4) is easy to state and holds for all symmetric spaces of the non-compact type. On the other hand, it is not parallel to the compact case and does not explicitly involve the geometry of the dual compact symmetric space. A different approach was developed by the authors of the current paper [24,25] in the case of a non-compact Riemannian symmetric space of the "complex type," that is, a space of the form $X=G / K$ where $G$ is complex semisimple and $K$ is a maximal compact subgroup (see also [26] for analogous results on compact quotients of such symmetric spaces). The results of [24,25] are extremely parallel to Equations (1) and (2), with $v_{t}$ now representing an "unwrapped" version of the heat kernel on the dual compact symmetric space to $X$, except that there is a subtle cancellation of singularities that allows the formulas to make sense. When one moves away from the complex case, the singularities become more complicated, and the results of $[24,25]$ do not extend as stated.

In the present paper, we consider the case in which $X$ is an odd-dimensional hyperbolic space, $H^{2 n+1}$, in which case $\tilde{D}$ is a differential operator. As our first main goal, we develop an isometry formula that is as parallel as possible to the compact case. We do this by taking the adjoint (with respect to certain natural inner products) of the operator $\tilde{D}$ in Equation (4), which is done by integrating by parts. Two main observations allow us to construct an isometry formula similar to Equation (1).

- If $\tilde{D}^{*}$ is the adjoint of $\tilde{D}$, then $\tilde{D}^{*}\left(w_{t}\right)$ is an "unwrapped" version of the heat kernel for the dual compact symmetric space $S^{2 n+1}$.

- Taking the adjoint of $\tilde{D}$ involves boundary terms, which are meromorphic functions of the radius. These functions tend to zero as long as we avoid poles at integer multiples of $\pi$. 
As our second main goal, we develop an inversion formula. We begin by developing a general inversion formula, in the style of [20], that involves a shift operator and applies to an arbitrary symmetric space of the non-compact type. We then specialize to the case of odd-dimensional hyperbolic spaces, integrate by parts and obtain an inversion formula similar to Equation (2).

\section{Main Results}

We let $G / K=H^{2 n+1}$ denote the hyperbolic space of dimension $2 n+1$, with the metric normalized to have constant sectional curvature -1 , where $G$ is the identity component of $S O(2 n+1,1)$ and $K=S O(2 n+1)$. We refer to Section 5.7 of [27] for standard formulas involving the metric and the Laplacian on $H^{2 n+1}$. We consider the map from $T\left(H^{2 n+1}\right) \rightarrow G_{\mathbb{C}} / K_{\mathbb{C}}$ given by:

$$
(x, Y) \mapsto \exp _{x}(i Y),
$$

where the right-hand side of the above formula refers to the analytic continuation of the geometric exponential map. For all sufficiently small $R$, the map Equation (5) is a diffeomorphism of the set $\{(x, Y)|| Y \mid<R\}$ onto its image. We refer to the set of points of the form $\exp _{x}(i Y)$ with $|Y|<R$ as a tube in $G_{\mathbb{C}} / K_{\mathbb{C}}$.

If we analytically continue the metric tensor from $G / K=H^{2 n+1}$ to a tube in $G_{\mathbb{C}} / K_{\mathbb{C}}$ and then restrict to the image of a single fiber in $T(G / K)$, the result is the negative of a Riemannian metric ([28] Proposition 1.17). Under this metric, the fibers are locally isometric to the unit sphere $S^{2 n+1}$. We begin by introducing the relevant density to be used in the fibers.

Definition 1. For each nonnegative integer $n$ and $t, r \in \mathbb{R}$ with $t>0$, let $\nu_{t}(r)$ be given by:

$$
\nu_{t}(r)=e^{t n^{2} / 2}\left(-\frac{1}{2 \pi} \frac{1}{\sin r} \frac{d}{d r}\right)^{n} \frac{e^{-r^{2} /(2 t)}}{\sqrt{2 \pi t}} .
$$

We refer to this function as the unwrapped heat kernel for $S^{2 n+1}$. The $2 \pi$-periodization of this function with respect to $r$ is the actual heat kernel on $S^{2 n+1}$ (compare Proposition 7). The unwrapped heat kernel is nonsingular at the origin, but has a pole of order $2 n-1$ at nonzero integer multiples of $\pi$. Our results are based on the idea of taking a limit that "stays a fixed distance away from the poles."

Notation 2. A limit as $R \rightarrow+\infty$ staying a fixed distance from the poles means a limit as $R$ e $R$ tends to $+\infty$ in a region of the form:

$$
S_{\varepsilon, A}=\{R \in \mathbb{C}|\operatorname{Re} R>0,| \operatorname{Im} R|<A,| R-n \pi \mid>\varepsilon, n=1,2,3, \ldots\},
$$

where $0<\varepsilon<A<\pi$. See Figure 1 .

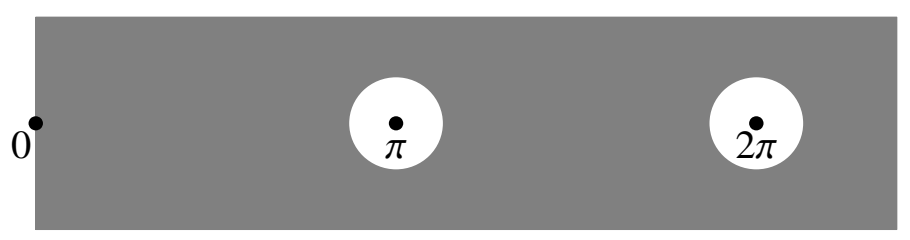

Figure 1. A typical domain of the form $S_{\varepsilon, A}$. 
With our notation established, we are ready to state our first main result.

Theorem 3 (Isometry theorem). Fix $f \in L^{2}\left(H^{2 n+1}\right)$ and let $F=\left(e^{t \Delta / 2} f\right)_{\mathbb{C}}$. Then, for all sufficiently small $R$, the integral:

$$
I(R)=\int_{x \in H^{2 n+1}} \int_{\substack{Y \in T_{x}\left(H^{2 n+1}\right) \\|Y| \leq R}}\left|F\left(\exp _{x}(i Y / 2)\right)\right|^{2} \nu_{2 t}(|Y|) \frac{\sin ^{2 n}(|Y|)}{|Y|^{2 n}} d Y d x
$$

is defined and convergent. Furthermore, $I(R)$ extends to a meromorphic function on $\mathbb{C}$ with poles only at nonzero integer multiples of $\pi$, and:

$$
\|f\|_{L^{2}\left(H^{2 n+1}\right)}^{2}=\lim _{R \rightarrow+\infty} I(R),
$$

where the limit is taken staying a fixed distance from the poles.

The factor $\sin ^{2 n}(|Y|) /|Y|^{2 n}$ is just the hyperbolic version of the Jacobian factor $J(Y)$ in Equation (1). In this rank-one case, the unwrapped heat kernel $v_{2 t}$ depends only on $|Y|$. Since the identity component of $S O(3,1)$ admits a complex structure, the symmetric space $H^{3}$ belongs to the complex case. When $n=1$, the preceding result is a special case of Theorem 3 of [25], after making a change of variables by a factor of two in $Y$. In the $n=1$ case, however, the function $I(R)$ has no poles.

We emphasize that the initial definition of $I(R)$ in Equation (7) does not make sense for large $R$, because $F\left(\exp _{x}(i Y)\right)$ is only defined when $Y$ is in a certain bounded region $\Omega$, since $F:=\left(e^{t \Delta / 2} f\right)_{\mathbb{C}}$ does not extend to the whole complexification of $H^{2 n+1}$. Thus, the limit on the right-hand side of Equation (8) refers to the meromorphic extension of $I(R)$.

Now, since $H^{2 n+1}$ has rank one, the $G$-orbit through $\exp _{x_{0}}\left(i Y_{0} / 2\right)$ consists of all points of the form $\exp _{x}(i Y / 2)$, where $|Y|=\left|Y_{0}\right|$. Thus, we may think of the integral on the right-hand side of Equation (7) as an orbital integral through a point with $\left|Y_{0} / 2\right|=R$, followed by integration with respect to $R$ (see the first several paragraphs of Section 5). Since, as we have noted, $\nu_{t}(r)$ has a pole of order $2 n-1$ at each nonzero (integer) multiple of $\pi$, we see that the density in Equation (7) has a zero of order one at each nonzero multiple of $\pi$. Except when $n=1$, however, this zero in the density is not sufficient to cancel out the singularities in the orbital integrals.

It may seem surprising that integration of the singular orbital integral of $F$ does not produce branching behavior in the function $I(R)$. The absence of branching is demonstrated by integrating by parts to convert $I(R)$ into a truncated version of the isometry formula in Equation (4). Since the integrand on the right-hand side of Equation (4) is nonsingular, the only singularities in $I(R)$ will come from the boundary terms, which do not involve integration.

We turn, next, to the development of an inversion formula. We begin with an inversion formula in the style of [20], which involves the shift operator and applies to an arbitrary symmetric space of the non-compact type. We then specialize this formula to the case of an odd-dimensional hyperbolic space and perform an integration by parts, yielding a formula that is similar to Equation (7).

Let $G$ be a non-compact semisimple Lie group, assumed to be connected and with finite center. Let $K$ be a maximal compact subgroup of $G$. The quotient $G / K$ has a $G$-invariant metric making $G / K$ into a Riemannian symmetric space of the non-compact type. For each point $x$ in $G / K$, let $K_{x}$ denote the 
stabilizer of $x$ in $G$, so that $K_{x}$ is conjugate to $K$ in $G$. Then, let $f^{(x)}$ denote the average of $f$ over the action of $K_{x}$; that is,

$$
f^{(x)}(y)=\int_{K_{x}} f(g \cdot y) d g
$$

where $d g$ is the normalized Haar measure on $K_{x}$. If $F=e^{t \Delta / 2} f$, then since the heat operator commutes with all isometries of $G / K$, we can say that $F^{(x)}$ may be computed either as the average of $F$ over the action of $K_{x}$ or as the heat operator applied to $f^{(x)}$. Since $F^{(x)}$ is invariant under the action of $K_{x}$, the function:

$$
Y \mapsto F^{(x)}\left(\exp _{x}(Y)\right)
$$

is determined by its values on the tangent space $\mathfrak{a}_{x}$ to a maximal flat through $x$.

We let $H^{r}(G / K)$, where $r$ is a positive real number, denote the Sobolev space of functions on $G / K$ "having $r$ derivatives in $L^{2}$." More precisely, consider the positive operator $I-\Delta$, viewed as an unbounded self-adjoint operator on $L^{2}(G / K)$. We then take $H^{r}(G / K)$ to be the domain of the operator $(I-\Delta)^{r / 2}$, where $(I-\Delta)^{r / 2}$ is defined by the functional calculus for self-adjoint operators. In Section 7, we will describe the Sobolev space $H^{r}(G / K)$ more concretely in terms of the Helgason Fourier transform for $G / K$.

Theorem 4 (Inversion formula for an arbitrary symmetric space). Fix $f \in L^{2}(G / K)$, and let $F=e^{t \Delta / 2} f$. There is a positive constant $r$ (depending only on $G$ ), such that if $f$ belongs to the Sobolev space $H^{r}(G / K)$, we have:

$$
f(x)=e^{t|\delta|^{2} / 2} \int_{\mathfrak{a}_{x}} D\left(F^{(x)}\right)\left(\exp _{x}(i Y)\right) \frac{e^{-|Y|^{2} /(2 t)}}{(2 \pi t)^{k / 2}} d Y,
$$

with absolute convergence of the integral. Here, D is the shift operator defined in Section 3.2 of [20].

Upon specializing Theorem 4 to the case of $H^{2 n+1}$, we may integrate by parts, with appropriate attention to the boundary terms, to obtain an inversion formula similar to the isometry formula in Theorem 3. As always, there is a factor of two difference in the scaling of the variables between the isometry formula and the inversion formula.

Theorem 5 (Inversion formula for $\left.H^{2 n+1}\right)$. Fix $f \in L^{2}\left(H^{2 n+1}\right)$, and let $F=e^{t \Delta / 2} f$. Define, for all sufficiently small $R$,

$$
J(R, x)=\int_{\substack{Y \in T_{x}\left(H^{2 n+1}\right) \\|Y| \leq R}} F\left(\exp _{x}(i Y)\right) \nu_{t}(|Y|) \frac{\sin ^{2 n}(|Y|)}{|Y|^{2 n}} d Y .
$$

Then, for each $x \in H^{2 n+1}$, the function $J(R, x)$ extends to a meromorphic function on $\mathbb{C}$ with poles only at nonzero integer multiples of $\pi$. Furthermore, there is a positive constant $r$ (depending only on $n$ ), such that if $f$ belongs to the Sobolev space $H^{r}\left(H^{2 n+1}\right)$, we have:

$$
f(x)=\lim _{R \rightarrow+\infty} J(R, x)
$$

where the limit is taken along a path in $\mathbb{C}$ that stays a fixed distance away from the poles. 
The $n=1$ case of the preceding theorem is a special case of Theorem 6 in [24]. In the $n=1$ case, the function $J(R, x)$ actually has no poles.

We now turn to the question of a surjectivity theorem. In the complex case, we proved that if $F$ is a holomorphic function for which $I(R)$ is defined for small $R$ and has an analytic continuation with finite limit at $+\infty$, then $F=\left(e^{t \Delta / 2} f\right)_{\mathbb{C}}$ for some $f \in L^{2}(G / K)$. For technical reasons that will be discussed in Section 6, it seems difficult to prove such a result in any case other than the complex case. For the case of $H^{2 n+1}, n \geq 2$, we content ourselves with the following weaker result. In the following result, we make use of the Helgason-Fourier transform, as described in Section 2.3 of [20]. If $f$ is a function on $H^{2 n+1}=G / K$, the Fourier transform of $f$, denoted $\hat{f}$, is a function on $\mathfrak{a}^{*} \times B$, where $\mathfrak{a} \cong \mathbb{R}$ is the tangent space to a maximal flat in $H^{2 n+1}$ and where $B=M \backslash K$, with $M$ being the centralizer of $\mathfrak{a}$ in $K$.

Theorem 6. Suppose $F$ is a holomorphic function on a tube for which $I(R)$ is defined for all sufficiently small $R$. Then, the restriction of $F$ to $H^{2 n+1}$ is square integrable. Let $F_{\varepsilon}$ denote the function whose Fourier transform is given by:

$$
\hat{F}_{\varepsilon}(\lambda, b)=\hat{F}(\lambda, b) \mathbf{1}_{\{|\lambda|<1 / \varepsilon\}}
$$

and let $I\left(R ; F_{\varepsilon}\right)$ denote the quantity in Equation $(7)$ with $F$ replaced by $F_{\varepsilon}$. If:

$$
\lim _{\varepsilon \rightarrow 0} \lim _{R \rightarrow \infty} I\left(R ; F_{\varepsilon}\right)
$$

exists and is finite, there exists $f \in L^{2}\left(H^{2 n+1}\right)$, such that $F=\left(e^{t \Delta / 2} f\right)_{\mathbb{C}}$.

\section{Remarks on the Proofs of the Main Results}

At a fundamental level, the isometry and inversion formulas are based on a duality between the geometry of the base, $G / K=H^{2 n+1}$, and the geometry of the fibers in the local identification of $T(G / K)$ with $G_{\mathbb{C}} / K_{\mathbb{C}}$. As we have noted ([28] Proposition 1.17), the analytic continuation of the metric from the base restricts to the negative of a Riemannian metric on a neighborhood of the identity in the fibers. The fibers, with the resulting metric, are locally isometric to the dual compact symmetric space $S^{2 n+1}$. For holomorphic functions on a tube in $G_{\mathbb{C}} / K_{\mathbb{C}}$, we have the following key result:

$$
\begin{aligned}
& \text { (spherical Laplacian in fibers) } \\
& =-(\text { hyperbolic Laplacian in base })
\end{aligned}
$$

(Compare [28] Proposition 1.19.) This fundamental identity is ultimately responsible for all of our main results. It follows formally from Equation (9), for example, that doing the forward heat operator in the fibers accomplishes the backward heat operator for the base, which is precisely the inversion formula. When the base is compact, the appropriate version of the fundamental identity is the key to proving both the isometry and inversion formulas [6,16].

When the base is non-compact, a convenient way to exploit the fundamental identity is to use spherical functions. In the case of the isometry formula, for example, there is a Gutzmer-type formula due to Faraut $[21,29]$, which says that the orbital integral in Equation (3) may be computed as:

$$
\mathcal{O}_{|F|^{2}}(i r)=\int_{\mathbb{R}}\|\hat{f}(\lambda)\|^{2} e^{-t\left(|\lambda|^{2}+|\delta|^{2}\right)} \phi_{\lambda}(i r) d \mu(\lambda),
$$


where $\|\hat{f}(\lambda)\|$ is the $L^{2}$ norm of the Fourier transform $\hat{f}(\lambda, b)$ of $f$ over the $b$ variable and where $\phi_{\lambda}$ is the spherical function with parameter $\lambda \in \mathbb{R}$. Now, as discussed in Section 5, an appropriate integral of $\mathcal{O}_{|F|^{2}}(i r)$ over $r$ gives the quantity $I(R)$ in the statement of Theorem 3. Thus, Equation (10) becomes:

$$
\begin{aligned}
I(R) & =c_{n} \int_{0}^{R} \mathcal{O}_{|F|^{2}}(i r) \nu_{2 t}(r) \sin ^{2 n} r d r \\
& =\int_{\mathbb{R}}\|\hat{f}(\lambda)\|^{2} e^{-t\left(|\lambda|^{2}+|\delta|^{2}\right)}\left[c_{n} \int_{0}^{R} \phi_{\lambda}(i r) \nu_{2 t}(r) \sin ^{2 n} r d r\right] d \mu(\lambda) .
\end{aligned}
$$

Meanwhile, $\phi_{\lambda}(r)$ is the restriction to a maximal flat of a radial-type eigenfunction for the Laplacian for $H^{2 n+1}$. Thus, by Equation (9), $\phi_{\lambda}(i r)$ is the restriction to a maximal flat of a radial-type eigenfunction for the Laplacian for $S^{2 n+1}$. The expression in square brackets on the right-hand side of Equation (11) is then a polar-coordinates computation of the integral of this eigenfunction against the unwrapped spherical heat kernel $v_{2 t}$.

Now, we expect that the integral of an eigenfunction of the Laplacian against the heat kernel $\nu_{2 t}$ should give $e^{t \lambda}$ times the value of the eigenfunction at the base point, which is one in the case of a spherical function. Since $\phi_{\lambda}(r)$ has eigenvalue $-\left(|\lambda|^{2}+|\delta|^{2}\right)$ for the hyperbolic Laplacian, $\phi_{\lambda}(i r)$ has eigenvalue $|\lambda|^{2}+|\delta|^{2}$ for the spherical Laplacian. We expect, then, that:

$$
\lim _{R \rightarrow+\infty} c_{n} \int_{0}^{R} \phi_{\lambda}(i r) v_{2 t}(r) \sin ^{2 n} r d r=e^{t\left(|\lambda|^{2}+|\delta|^{2}\right)} .
$$

Thus, formally, letting $R$ tend to infinity in Equation (11) should give:

$$
\begin{aligned}
\lim _{R \rightarrow \infty} I(R) & =\int_{\mathbb{R}}\|\hat{f}(\lambda)\|^{2} e^{-t\left(|\lambda|^{2}+|\delta|^{2}\right)} e^{t\left(|\lambda|^{2}+|\delta|^{2}\right)} d \mu(\lambda) \\
& =\|f\|^{2}
\end{aligned}
$$

which is our isometry formula. A similar formal analysis yields the inversion formula (start with Equation (37) in Section 7 and apply Equation (12) with $t$ replaced by $t / 2$ ).

Of course, the preceding analysis is only formal, since it takes no account of the singularities involved. In the case of odd-dimensional hyperbolic spaces, the analytically continued spherical function $\phi_{\lambda}(\mathrm{ir})$ has singularities at nonzero integer multiples of $\pi$. The claim is that $\phi_{\lambda}(i r)$ is actually meromorphic and that the above analysis can be made rigorous if we simply integrate along a contour that avoids the poles.

To establish this claim, it is convenient to make use of shift operators. The unwrapped heat kernel $v_{2 t}$ can be expressed as a certain shift operator applied to a Gaussian. Repeated integrations by parts move the shift operator off the Gaussian and onto the spherical function, where it changes $\phi_{\lambda}$ into its Euclidean counterpart, $\cosh (\lambda r)$ (see Section 4). Thus, after integrating by parts a finite number of times, we end up with an integral (of a Gaussian times $\cosh (\lambda r)$ ) that has no singularities and whose value can be computed explicitly. The boundary terms in the integration by parts are manifestly meromorphic, and they tend to zero as $R$ tends to infinity, thus leading to a rigorous version of Equation (12).

To prove the isometry formula, it still remains to interchange the limit as $R$ tends to infinity with the integral on the right-hand side of Equation (11). Justifying this interchange for a general square-integrable function $f$ requires sharp estimates on the analytically continued spherical functions and their derivatives, which we obtain in Section 4. 
For any symmetric space of the non-compact type, an analog of the fundamental identity Equation (9) holds [28]. If one can construct a suitable unwrapped heat kernel and prove an analog of Equation (12), one may hope to prove isometry and inversion formulas along the lines of what we have done here for $H^{2 n+1}$.

\section{Shift Operators and Spherical Functions}

In this section, we consider various sorts of shift operators, each of which has an "intertwining property" relating the radial part of a non-Euclidean Laplacian (for $H^{2 n+1}$ or $S^{2 n+1}$ ) to the Euclidean Laplacian for $\mathbb{R}^{1}$. There are a total of four shift operators, two that shift (in one direction or the other) between $H^{2 n+1}$ and $\mathbb{R}^{1}$ and two that shift between $S^{2 n+1}$ and $\mathbb{R}^{1}$. We also describe how the shift operators act on spherical functions and use the resulting formulas to derive estimates on the spherical functions and their derivatives.

Our first two shift operators are defined as follows:

$$
\begin{aligned}
D^{*} & =\left(-\frac{1}{2 \pi} \frac{1}{\sinh r} \frac{d}{d r}\right)^{n} \\
\tilde{D}^{*} & =\left(-\frac{1}{2 \pi} \frac{1}{\sin r} \frac{d}{d r}\right)^{n} .
\end{aligned}
$$

The star in the notation indicates that these operators are the adjoints of other shift operators, which we will introduce shortly. It is known (e.g., [30] p. 316) and not hard to verify by direct calculation that these shift operators have the following intertwining properties:

$$
\begin{aligned}
\left(\frac{d^{2}}{d r^{2}}+2 n \frac{\cosh r}{\sinh r} \frac{d}{d r}\right) D^{*} & =D^{*}\left(\frac{d^{2}}{d r^{2}}-n^{2}\right) \\
\left(\frac{d^{2}}{d r^{2}}+2 n \frac{\cos r}{\sin r} \frac{d}{d r}\right) \tilde{D}^{*} & =\tilde{D}^{*}\left(\frac{d^{2}}{d r^{2}}+n^{2}\right) .
\end{aligned}
$$

The operators in parentheses on the left-hand sides are the radial parts of the Laplacians for $H^{2 n+1}$ and $S^{2 n+1}$, respectively.

The operators $D^{*}$ and $\tilde{D}^{*}$ may be used to convert the heat kernels for $\mathbb{R}$ and for $S^{1}$ into the heat kernels for $H^{2 n+1}$ and $S^{2 n+1}$, respectively. For us, the term "heat kernel" will always refer to the fundamental solution of the heat equation:

$$
\frac{\partial u}{\partial t}=\frac{1}{2} \Delta u
$$

where $\Delta$ is the Laplacian, which we take to be a negative operator.

Proposition 7. The heat kernel $\gamma_{t}$ on $H^{2 n+1}$ may be computed as:

$$
\gamma_{t}(r)=e^{-t n^{2} / 2} D^{*}\left(\frac{1}{\sqrt{2 \pi t}} e^{-r^{2} /(2 t)}\right)
$$

and the heat kernel $\rho_{t}$ on $S^{2 n+1}$ may be computed as:

$$
\rho_{t}(r)=e^{t n^{2} / 2} \tilde{D}^{*}\left(\frac{1}{\sqrt{2 \pi t}} \sum_{k=-\infty}^{\infty} e^{-(r-2 \pi k)^{2} /(2 t)}\right),
$$

where $r$ denotes the geodesic distance from the base point. 
By comparing the above expression for $\rho_{t}$ to the definition of the unwrapped heat kernel $v_{t}$ (Definition 1) and by noting that $\tilde{D}^{*}$ commutes with translations by $2 \pi$, we see that the $2 \pi$-periodization of $\nu_{t}$ is simply $\rho_{t}$. This observation justifies the term "unwrapped heat kernel" for $\nu_{t}$.

Proof. Although this result is known ([31] Section 8), we briefly outline the proof. It follows from Equations (15) and (16) that both $\gamma_{t}$ and $\rho_{t}$ satisfy the heat equation. It remains only to show that these are the fundamental solutions. Clearly, both $\gamma_{t}$ and $\rho_{t}$ will decay away from the base point, and near the base point, the shift operators behave much like:

$$
\frac{1}{(2 \pi)^{n}}\left(-\frac{1}{r} \frac{d}{d r}\right)^{n},
$$

which, by explicit computation, takes $(2 \pi t)^{-1 / 2} e^{-r^{2} /(2 t)}$ to $(2 \pi t)^{-(2 n+1) / 2} e^{-r^{2} /(2 t)}$. Thus, for small $t$, both $\gamma_{t}$ and $\rho_{t}$ look like the heat kernel for $\mathbb{R}^{2 n+1}$, which in turn behaves like a $\delta$-function.

The formulas for the integral of a radial function on $H^{2 n+1}$ and $S^{2 n+1}$ take the form:

$$
\begin{aligned}
& c_{n} \int_{0}^{\infty} f(r) \sinh ^{2 n} r d r \\
& c_{n} \int_{0}^{\pi} f(r) \sin ^{2 n} r d r,
\end{aligned}
$$

respectively, where $r$ is the geodesic distance from the base point and where:

$$
c_{n}:=\frac{2(2 \pi)^{n}}{(2 n-1) ! !}
$$

is the surface area of the unit sphere in $\mathbb{R}^{2 n+1}$. We now regard $D^{*}$ and $\tilde{D}^{*}$ as maps from an $L^{2}$ space with Lebesgue measure to a $L^{2}$ space with the measures coming from polar coordinates. We then compute the adjoints of these maps (modulo boundary terms), which we call $D$ and $\tilde{D}$, respectively.

Proposition 8. Let:

$$
\begin{aligned}
& D=\frac{1}{(2 n-1) ! !} \prod_{k=1}^{n}\left(\sinh r \frac{d}{d r}+(2 k-1) \cosh r\right) \\
& \tilde{D}=\frac{1}{(2 n-1) ! !} \prod_{k=1}^{n}\left(\sin r \frac{d}{d r}+(2 k-1) \cos r\right),
\end{aligned}
$$

where the product is taken with smaller values of $k$ to the left and larger values of $k$ to the right. Then, for all sufficiently smooth even functions $f$ and $g$ on $[-R, R]$, we have:

$$
\begin{aligned}
& 2 \int_{0}^{R}(D f)(r) g(r) d r=\text { B.T. }+c_{n} \int_{0}^{R} f(r)\left(D^{*} g\right)(r) \sinh ^{2 n} r d r \\
& 2 \int_{0}^{R}(\tilde{D} f)(r) g(r) d r=\text { B.T. }+c_{n} \int_{0}^{R} f(r)\left(\tilde{D}^{*} g\right)(r) \sin ^{2 n} r d r,
\end{aligned}
$$

where "B.T." indicates boundary terms that involve the values of $f$ and $g$ and their derivatives at $R$. Furthermore, $D$ and $\tilde{D}$ have the following intertwining properties:

$$
\begin{aligned}
D\left(\frac{d^{2}}{d r^{2}}+2 n \frac{\cosh r}{\sinh r} \frac{d}{d r}\right) & =\left(\frac{d^{2}}{d r^{2}}-n^{2}\right) D \\
\tilde{D}\left(\frac{d^{2}}{d r^{2}}+2 n \frac{\cos r}{\sin r} \frac{d}{d r}\right) & =\left(\frac{d^{2}}{d r^{2}}+n^{2}\right) \tilde{D} .
\end{aligned}
$$


We emphasize that the boundary terms do not involve the values of the functions or their derivatives at zero, but only at $R$.

Proof. If $g$ is smooth and even on $[-R, R]$, then $g^{\prime}(r) / \sinh r$ has a removable singularity at $r=0$, and the resulting function is again smooth and even on $[-R, R]$. Thus, there are no singularities in the computation of $D^{*} g$, despite the factors of $\sinh r$ in the denominator in the definition of $D^{*}$ and similarly for $\tilde{D}^{*} g$.

We start on the right-hand side of, say, Equation (17) and successively integrate by parts to move each factor in the definition of $D^{*}$ off of $g$ and onto $f$. To this end, we compute that:

$$
\begin{aligned}
& c_{k+1} \int_{0}^{R} f(r)\left[\left(-\frac{1}{2 \pi} \frac{1}{\sinh r} \frac{d}{d r}\right) g(r)\right] \sinh ^{2(k+1)} r d r \\
& =\text { B.T. }+\frac{c_{k}}{2 k+1} \int_{0}^{R}\left[\sinh r f^{\prime}(r)+(2 k+1) \cosh r f(r)\right] g(r) \sinh ^{2 k} r d r,
\end{aligned}
$$

where the boundary term comes from evaluating:

$$
-\left(c_{k+1} /(2 \pi)\right) \sinh ^{2 k+1} r f(r) g(r)
$$

at zero and at $R$ and where $c_{k+1} /(2 \pi)=c_{k} /(2 k+1)$. Note that even if $k=0$, the boundary term at zero vanishes. Integrating by parts $n$ times then yields the first claimed identity, and an entirely similar calculation verifies the second identity. Finally, since the radial part of each Laplacian is a symmetric operator on a dense subspace of its respective Hilbert space, Equations (19) and (20) follow from Equations (15) and (16) by taking adjoints (we may initially prove the desired identities on $C_{c}^{\infty}((0, \infty))$ and then extend to general smooth functions by using the local nature of all operators involved).

Lemma 9. For any sufficiently nice function $f$, we have:

$$
(D f)(0)=f(0) \text {. }
$$

Proof. Although it is easy enough to verify this claim directly from the formula for $D$, it is more illuminating to use Equation (17). If we apply Equation (17) with $g$ equal to the Euclidean heat kernel at time $t$, then $D^{*} g$ is the heat kernel at time $t$ for $H^{2 n+1}$ multiplied by the constant $a_{n}(t):=e^{t n^{2} / 2}$. Then, the integral on the right-hand side of Equations (17) is the integral of the radial extension of $f$ against the heat kernel on $H^{2 n+1}$ multiplied by $a_{n}(t)$, while the left-hand side is the integral of the even extension of $D f$ against the heat kernel on $\mathbb{R}$. Letting $t$ tend to zero gives the claimed result, as the boundary terms will vanish in the limit.

Proposition 10. The operator $D$ in Proposition 8 maps the spherical function $\phi_{\lambda}$ for $H^{2 n+1}$ to the function $\cos (\lambda r)$. The operator $\tilde{D}$ in Proposition 8 maps the analytically continued spherical function $\phi_{\lambda}($ ir $)$ to the function $\cosh (\lambda r)$.

Proof. The spherical function $\phi_{\lambda}$ is an even eigenfunction for the radial part of the hyperbolic Laplacian with eigenvalue $-\lambda^{2}-n^{2}$. Thus, by Equations (19), the function $D \phi_{\lambda}$ is an eigenfunction for $d^{2} / d r^{2}$ with eigenvalue $-\lambda^{2}$. Since, also, $D \phi_{\lambda}$ is even, we conclude that $D \phi_{\lambda}(r)$ is a constant multiple of $\cos (\lambda r)$. However, since $\phi_{\lambda}$ is normalized to equal one at the base point, Lemma 9 tells us that the constant is one. From the definition of $\tilde{D}$, we now see that $\tilde{D}$ maps the analytically continued spherical function $\phi_{\lambda}(i r)$ to $\left(D \phi_{\lambda}\right)(i r)$. That is, we have $\tilde{D}\left(\phi_{\lambda}(i r)\right)=\cosh (\lambda r)$, the Euclidean counterpart of $\phi_{\lambda}(i r)$. 
We next show that the adjoint operator $\tilde{D}^{*}$ maps $\cosh (\lambda r)$ to a constant (depending on $\lambda$ ) times $\phi_{\lambda}(i r)$.

Lemma 11. The analytically continued spherical function $\phi_{\lambda}(\mathrm{ir})$ may be obtained from its Euclidean counterpart by the action of $\tilde{D}^{*}$ as follows:

$$
\tilde{D}^{*}(\cosh (\lambda r))=c_{\lambda, n} \phi_{\lambda}(i r)
$$

where:

$$
c_{\lambda, n}=\frac{1}{(-2 \pi)^{n}} \prod_{k=0}^{n-1} \frac{\lambda^{2}+k^{2}}{2 k+1} .
$$

Proof. We let $\phi_{\lambda, k}$ denote the spherical function for $H^{2 k+1}$, so that $\phi_{\lambda, 0}(i r)=\cosh (\lambda r)$. We then claim that:

$$
\frac{1}{\sin r} \frac{d}{d r} \phi_{\lambda, k}(i r)=d_{\lambda, k} \phi_{\lambda, k+1}(i r)
$$

where:

$$
d_{\lambda, k}=\frac{\lambda^{2}+k^{2}}{2 k+1}
$$

To see this, let:

$$
\Delta_{k}:=\frac{d^{2}}{d r^{2}}+2 k \frac{\cos r}{\sin r} \frac{d}{d r}
$$

denote the radial part of the Laplacian on $S^{2 k+1}$. Direct calculation shows:

$$
\Delta_{k+1}\left(\frac{1}{\sin r} \frac{d}{d r}\right)-\left(\frac{1}{\sin r} \frac{d}{d r}\right) \Delta_{k}=(2 k+1)\left(\frac{1}{\sin r} \frac{d}{d r}\right)
$$

as operators on smooth functions of $r$. Since $\phi_{\lambda, k}(r)$ is an eigenfunction of the radial part of the Laplacian on $H^{2 k+1}$ with eigenvalue $-\lambda^{2}-k^{2}$, the function $\phi_{\lambda, k}(i r)$ is an eigenfunction of $\Delta_{k}$ with eigenvalue $\lambda^{2}+k^{2}$.

Consequently,

$$
\begin{aligned}
\Delta_{k+1}\left(\frac{1}{\sin r} \frac{d}{d r} \phi_{\lambda, k}(i r)\right) & =\left(\frac{1}{\sin r} \frac{d}{d r}\right) \Delta_{k}\left(\phi_{\lambda, k}(i r)\right)+(2 k+1)\left(\frac{1}{\sin r} \frac{d}{d r} \phi_{\lambda, k}(i r)\right) \\
& =\left(\lambda^{2}+(k+1)^{2}\right)\left(\frac{1}{\sin r} \frac{d}{d r} \phi_{\lambda, k}(i r)\right) .
\end{aligned}
$$

Both sides of Equation (22) therefore are even, real-analytic eigenfunctions for the radial part of the Laplacian on $S^{2 k+3}$ with the same eigenvalue. Thus, the two functions $\frac{1}{\sin r} \frac{d}{d r} \phi_{\lambda, k}(i r)$ and $\phi_{\lambda, k+1}(i r)$ are equal up to a constant, as claimed in Equation (22) (it is easy to check that the eigenfunction equation has exactly one series solution in even powers of $r$ ). To evaluate the constant, we let $r$ tend to zero on both sides of Equation (22), which gives, by L'Hospital's rule,

$$
\phi_{\lambda, k}^{\prime \prime}(0)=d_{\lambda, k} \phi_{\lambda, k+1}(0)=d_{\lambda, k}
$$

Meanwhile, $\phi_{\lambda, k}(0)=1$, and $\phi_{\lambda, k}(i r)$ satisfies:

$$
\frac{d^{2} \phi_{\lambda, k}(i r)}{d r^{2}}+2 k \frac{\cos r}{\sin r} \frac{d \phi_{\lambda, k}(i r)}{d r}=\left(\lambda^{2}+k^{2}\right) \phi_{\lambda, k}(i r) .
$$


Letting $r$ tend to zero in Equation (23) and using L'Hospital's rule again gives:

$$
(2 k+1) \phi_{\lambda, k}^{\prime \prime}(0)=\left(\lambda^{2}+k^{2}\right),
$$

which means that:

$$
d_{\lambda, k}=\phi_{\lambda, k}^{\prime \prime}(0)=\frac{\lambda^{2}+k^{2}}{2 k+1}
$$

as claimed. If we apply Equation (22) $n$ times and recall the definition of $\tilde{D}^{*}$ in Equation (14), we obtain the lemma.

We now estimate the analytically continued spherical function and its derivatives. In what follows, we do not require that $r$ be real, but allow it to range over a region of the form $S_{\varepsilon, A}$.

Lemma 12. For $r$ in the region $S_{\varepsilon, A}$ (Notation 2), the analytically continued spherical functions satisfy the following estimate:

$$
\left|\left(\frac{d}{d r}\right)^{l} \phi_{\lambda}(i r)\right| \leq C_{n, l} \frac{1+|r|}{(1+|\lambda|)^{n-l-1}} \frac{e^{|\lambda r|}-1}{|\lambda r|} .
$$

In particular, for $|\lambda|>1$ and $|r|>1$, we have:

$$
\left|\left(\frac{d}{d r}\right)^{l} \phi_{\lambda}(i r)\right| \leq D_{n, l} \frac{e^{|\lambda r|}}{|\lambda|^{n-l}}
$$

and for $|\lambda| \leq 1$, we have:

$$
\left|\left(\frac{d}{d r}\right)^{l} \phi_{\lambda}(i r)\right| \leq E_{n, l}(1+|r|) e^{r} .
$$

Here, $C_{n, l}, D_{n, l}$ and $E_{n, l}$ are constants that depend only on $n, l$ and $\varepsilon$.

Proof. As in the proof of Lemma 11, we let $\phi_{\lambda, k}$ denote the spherical function with parameter $\lambda$ for $H^{2 k+1}$. We note that, by Equation (22),

$$
\begin{aligned}
\phi_{\lambda, 1}(i r) & =\frac{1}{\lambda^{2}} \frac{1}{\sin r} \frac{d}{d r} \cosh (\lambda r) \\
& =\frac{\sinh (\lambda r)}{\lambda r} \frac{r}{\sin r} .
\end{aligned}
$$

Now, an elementary power-series argument shows that for all $l \geq 0$, we have the estimate:

$$
\left|\left(\frac{d}{d x}\right)^{l} \frac{\sinh x}{x}\right| \leq \frac{e^{|x|}-1}{|x|}
$$

so that:

$$
\begin{aligned}
\left|\left(\frac{d}{d r}\right)^{l} \frac{\sinh \lambda r}{\lambda r}\right| & \leq|\lambda|^{l} \frac{e^{|\lambda r|}-1}{|\lambda r|} \\
& \leq(1+|\lambda|)^{l} \frac{e^{|\lambda r|}-1}{|\lambda r|}
\end{aligned}
$$


We can now see inductively that $\phi_{\lambda, k}$ will be a finite linear combination of terms of the form:

$$
d_{\lambda, k}\left[\left(\frac{d}{d r}\right)^{l} \frac{\sinh (\lambda r)}{\lambda r}\right] r^{a} g(r)
$$

where:

$$
d_{\lambda, k} \leq \frac{C}{(1+|\lambda|)^{2 k-2}}
$$

where $a$ is either zero or one and where $g(r)$ is a rational expression in $\sin r$ and $\cos r$, with only sine factors in the denominator. In the region $S_{\varepsilon, A}$, the function $g(r)$ will be bounded. Thus, by Equation (27), we obtain the desired estimate.

We conclude this section with a simple estimate that will be used in both Sections 5 and 7.

Lemma 13. For any positive number a and positive integer $m$, there is a constant $C$, such that for all $\lambda>0$, we have:

$$
\sup _{R}\left|e^{\lambda R} R^{m} e^{-a R^{2} / 2}\right| \leq C\left(1+\lambda^{m}\right) e^{\lambda^{2} /(2 a)},
$$

where the supremum is taken over $R$ in a region of the form $S_{\varepsilon, A}$ (Notation 2).

Proof. By completing the square and writing $R^{m}=(R-\lambda / a+\lambda / a)^{m}$, we obtain:

$$
e^{\lambda R} R^{m} e^{-a R^{2} / 2}=e^{\lambda^{2} /(2 a)} e^{-a(R-\lambda / a)^{2} / 2} \sum_{l=0}^{m}\left(\begin{array}{c}
m \\
l
\end{array}\right)(R-\lambda / a)^{l}(\lambda / a)^{m-l} .
$$

Letting $x=R-\lambda / a$ and noting that the function $z \mapsto z^{l} e^{-a z^{2} / 2}$ is bounded on $S_{\varepsilon, A}$, we easily obtain the desired bound.

\section{The Isometry Formula}

In this section, we provide the proof of Theorem 3. In simple terms, the theorem follows from the isometry result of Krötz-Ólafsson-Stanton [20] by taking the adjoint of the operator $\tilde{D}$, which is done by means of integration by parts. Some effort, however, is required to show that the boundary terms in the integration by parts can be neglected.

Using Equation (18) in Proposition 8, the $H^{2 n+1}$ case of the isometry formula in Equation (4) becomes, after taking into account the symmetry of the orbital integral,

$$
\begin{aligned}
\|f\|^{2} & =\lim _{R \rightarrow \infty} 2 \int_{0}^{R} \tilde{D}\left[\mathcal{O}_{|F|^{2}}(i r)\right] w_{t}(r) d r \\
& =\lim _{R \rightarrow \infty}\left(\text { B.T. }+c_{n} \int_{0}^{R} \mathcal{O}_{|F|^{2}}(i r) \tilde{D}^{*}\left[w_{t}(r)\right] \sin ^{2 n} r d r\right) .
\end{aligned}
$$

Here, the orbital integral is computed using the group $G$ equal to the identity component of $S O(2 n+1,1)$. From the formulas for $w_{t}$ and $\tilde{D}^{*}$, we can see that $\tilde{D}^{*}\left[w_{t}(r)\right]$ coincides with $\nu_{2 t}(r)$, the unwrapped heat kernel at time $2 t$.

Recall that for small $R$, the map $(x, Y) \mapsto \exp _{x}(i Y)$ is a diffeomorphism of the set:

$$
\{(x, Y)|| Y \mid<R\} \subset T\left(H^{2 n+1}\right)
$$


onto its image in the complexification of $H^{2 n+1}$ and that the image of this diffeomorphism is called a tube. Since $H^{2 n+1}$ has rank one, each $G$-orbit in a tube will correspond under the diffeomorphism to a sphere bundle:

$$
\{(x, Y)|| Y \mid=a\} \subset T\left(H^{2 n+1}\right) .
$$

Now, in the definition of the orbital integral in Equation (3), the Haar measure $d g$ on $G$ should be normalized so that the pushforward of $d g$ to $G / K$ coincides with the volume measure on $G / K$ (that is to say, when $Y=0$, the orbital integral in Equation (3) should coincide with the $L^{2}$ norm of $F$ over $G / K)$. Meanwhile, each $G$-orbit carries a $G$-invariant volume form that is unique up to a constant. In a local trivialization of the bundle Equation (29), a $G$-invariant volume form may be constructed as a product of the volume form on the sphere and the volume form on the base. If the volume of the sphere is normalized to one, the resulting volume form will coincide with the integral against the Haar measure $d g$, where $d g$ is normalized as described above.

Therefore, if we compute the quantity $I(R)$ in the statement of Theorem 3 using polar coordinates, the integral over the sphere will simply be an orbital integral. Thus,

$$
I(R)=c_{n} \int_{0}^{R} \mathcal{O}_{|F|^{2}}(i r) \tilde{D}^{*}\left[w_{t}(r)\right] \sin ^{2 n} r d r,
$$

where we recognize the right-hand side of Equation (30) as the second term on the right-hand side of Equation (28). To prove Theorem 3, then, we need only show that the boundary term on the right-hand side of Equation (28) tends to zero as $R$ tends to infinity in $S_{\varepsilon, A}$.

To analyze the boundary terms, we use the Gutzmer-type formula of Faraut, which also plays a key role in the results of [20]. According to [21], the orbital integral may be computed as:

$$
\mathcal{O}_{|F|^{2}}(i r)=\int_{\mathbb{R}}\|\hat{f}(\lambda)\|^{2} e^{-t\left(|\lambda|^{2}+|\delta|^{2}\right)} \phi_{\lambda}(i r) d \mu(\lambda),
$$

where $\phi_{\lambda}$ is the spherical function with parameter $\lambda$. Here, $\hat{f}$ is the Fourier transform of $f$, viewed as a function $\mathfrak{a}^{*} \times B$, and $\|\hat{f}(\lambda)\|$ is the $L^{2}$ norm of $\hat{f}$ over $B$, with $\lambda \in \mathfrak{a}^{*}$ fixed. As usual, $\delta$ denotes half the sum of the positive roots with multiplicity, and we have used the action of the heat operator on the Fourier transform:

$$
\hat{F}(\lambda, b)=\hat{f}(\lambda, b) e^{-t\left(|\lambda|^{2}+|\delta|^{2}\right) / 2} .
$$

Finally, $\mu$ denotes a certain measure on $\mathfrak{a}^{*} \cong \mathbb{R}$, which may be computed in terms of the $c$-function (when $r=0$, Equation (31) just gives the Plancherel theorem, expressing the $L^{2}$ norm of $F$ over $H^{2 n+1}$ in terms of its Fourier transform).

The rapidly decaying factor of $e^{-t\left(|\lambda|^{2}+|\delta|^{2}\right)}$ in Equation (31) makes it easy to justify interchanging the operator $\tilde{D}$ with the integral, giving:

$$
\tilde{D}\left[\mathcal{O}_{|F|^{2}}(i r)\right]=\int_{\mathbb{R}}\|\hat{f}(\lambda)\|^{2} e^{-t\left(|\lambda|^{2}+|\delta|^{2}\right)} \tilde{D}\left[\phi_{\lambda}(i r)\right] d \mu(\lambda)
$$

Thus,

$$
\begin{aligned}
& 2 \int_{0}^{R} \tilde{D}\left[\mathcal{O}_{|F|^{2}}(i r)\right] w_{t}(r) d r \\
& =\int_{\mathbb{R}}\|\hat{f}(\lambda)\|^{2} e^{-t\left(|\lambda|^{2}+|\delta|^{2}\right)}\left[2 \int_{0}^{R} \tilde{D}\left[\phi_{\lambda}(i r)\right] w_{t}(r) d r\right] d \mu(\lambda) .
\end{aligned}
$$


On the other hand, using Equation (31) in the expression of $I(R)$ given in Equation (30) yields:

$$
I(R)=\int_{\mathbb{R}}\|\hat{f}(\lambda)\|^{2} e^{-t\left(|\lambda|^{2}+|\delta|^{2}\right)}\left[c_{n} \int_{0}^{R} \phi_{\lambda}(i r) \tilde{D}^{*}\left[w_{t}(r)\right] \sin ^{2 n}(r) d r\right] d \mu(\lambda) .
$$

By Equation (18) in Proposition 8, the right-hand side of Equation (32) and the right-hand side of Equation (33) differ just by a sequence of integrations by parts in the inner integral. Meanwhile, the quantity on the right-hand side of Equation (32) admits an entire analytic continuation as a function of $R$, since Proposition 10 confirms that $\tilde{D}\left[\phi_{\lambda}(i r)\right]$ is just the Euclidean spherical function $\cosh (\lambda r)$, which has no singularities. We must now analyze the boundary terms that arise if, say, we begin with Equation (33) and integrate by parts repeatedly in the inner integral. We need to show that each boundary term is a meromorphic function of $R$ and that these terms tend to zero if $R$ tends to infinity avoiding the poles.

To analyze the boundary terms, it is convenient to introduce the operator:

$$
L=-\frac{1}{2 \pi} \frac{1}{\sin r} \frac{d}{d r}, \text { so that } \tilde{D}^{*}=L^{n} .
$$

The boundary terms of the integral:

$$
\int_{0}^{R} \phi_{\lambda}(i r) \tilde{D}^{*}\left[w_{t}(r)\right] \sin ^{2 n}(r) d r
$$

are determined by successive integration by parts that each remove a power of $L$ from operation on $w_{t}$. Using induction and Equation (21) from the proof of Proposition 8, we find that each boundary term is a linear combination of terms of the form:

$$
\left.\left(\left(\frac{d}{d r}\right)^{l} \phi_{\lambda}(i r)\right)\right|_{r=R} \cdot \sin ^{j} R \cdot \cos ^{k} R \cdot\left(L^{m} w_{t}\right)(R),
$$

where $l+m \leq n-1$ and where all of the exponents $l, j, k$ and $m$ are non-negative integers. Note, however, that there are negative powers of $\sin r$ contained in the computation of $L^{m} w_{t}$. It is easy to see that the boundary terms tend to zero as $R \rightarrow \infty$ for each fixed $\lambda$; what requires some effort is to justify interchanging the limit as $R \rightarrow \infty$ with the integral over $\lambda$ in Equation (33).

Since the only singularities in each boundary term are the negative powers of $\sin r$ arising from $L^{m} w_{t}$, we see that the boundary terms are meromorphic with poles only at integer multiples of $\pi$. Furthermore, since $w_{t}(r)$ is an even function of $r$, it is straightforward to check by induction that $L^{m} w_{t}$ is even and nonsingular at the origin. Thus, the singularities are only at nonzero integer multiples of $\pi$.

Now, another application of induction confirms that $\left(L^{m} w_{t}\right)(R)$ is a linear combination of terms of the form:

$$
e^{-\frac{R^{2}}{4 t}} \cdot \sin ^{p} R \cdot \cos ^{q} R \cdot R^{d}
$$

where $d \leq m$. Here, the exponents $q$ and $d$ are positive, but the the exponent $p$ of $\sin R$ may now be negative. Within the region $S_{\varepsilon, A}$, positive powers of $\cos R$ and arbitrary powers of $\sin R$ are bounded. Thus, for $|\lambda| \geq 1$, we can apply Equation (25) of Lemma 12 to show that each contribution to each boundary term is bounded by:

$$
C \frac{e^{|\lambda r|}}{|\lambda|^{l}} R^{d} e^{-R^{2} /(4 t)}
$$


where $l+d \leq l+m \leq n-1$ and where $C$ is a constant, independent of $\lambda$ and $R$.

If we apply Lemma 13 with $a=1 /(2 t)$, we find that, for $|\lambda|>1$ and $R>1$, each term of the form Equation (35) is bounded by:

$$
C e^{t|\lambda|^{2}} / R \text {. }
$$

Meanwhile, for $|\lambda| \leq 1$, we may apply Equation (26) of Lemma 12 to bound Equation (35) by:

$$
C R^{s} e^{R} e^{-R^{2} /(4 t)}
$$

for some $s$. Since $\|\hat{f}(\lambda)\|^{2}$ is integrable as a function of $\lambda$, it now follows by dominated convergence that the boundary terms relating Equations (32) and (33) tend to zero as $\operatorname{Re} R \rightarrow+\infty$ in the region $S_{\varepsilon, A}$.

\section{The Surjectivity Theorem}

We would like to establish a result showing that if $F$ is any holomorphic function on a tube for which $I(R)$ has an analytic continuation with finite limit at infinity, then $F$ must be in the image of the heat operator. To approach such a result, we apply the Gutzmer formula of Faraut, which tells us that:

$$
I(R)=\int_{\mathbb{R}}\|\hat{F}(\lambda)\|^{2} c_{n} \int_{0}^{R} \phi_{\lambda}(i r) \tilde{D}^{*}\left[w_{t}(r)\right] \sin ^{2 n}(r) d r d \mu(\lambda) .
$$

If we could interchange the limit as $R$ tends to infinity (away from the poles) with the outer integral, we could apply the rigorous version of Equation (12) to conclude that the limit of $I(R)$ coincides with:

$$
\int_{\mathbb{R}}\|\hat{F}(\lambda)\|^{2} e^{t\left(|\lambda|^{2}+|\delta|^{2}\right)} d \mu(\lambda)
$$

If we had this result and the limit of $I(R)$ were finite, we would conclude that $F=e^{t \Delta / 2} f$, where $f$ is the function whose Fourier transform is defined by $\hat{f}(\lambda, b)=\hat{F}(\lambda, b) e^{-t\left(|\lambda|^{2}+|\delta|^{2}\right)}$.

In the complex case, the desired interchange of limit and integral can be justified by showing that the analog of the inner integral in Equation (36) is positive and monotone as a function of $R$ (see the inner integral on the right-hand side of Equation (37) in Theorem 6 of [25]). We thus obtain a "strong" surjectivity result in the complex case ([25] Theorem 8). In the case at hand, however, numerical calculations indicate that the quantity:

$$
c_{n} \int_{0}^{R} \phi_{\lambda}(i r) \tilde{D}^{*}\left[w_{t}(r)\right] \sin ^{2 n}(r) d r
$$

is neither positive nor monotone, except when $n=1$ (i.e., except in the $H^{3}$ case, when $G$ is complex).

We can still prove, however, a weaker form of the surjectivity theorem for $H^{2 n+1}$, as in Theorem 6.

Proof of Theorem 6. According to the results of [29], if $I(R)$ exists and is finite, the restriction of $F$ to $H^{2 n+1}$ is square-integrable. It therefore makes sense to construct the function $F_{\varepsilon}$. The function $F_{\varepsilon}$ is of the form $F_{\varepsilon}=e^{t \Delta / 2} f_{\varepsilon}$, where $f_{\varepsilon}$ is the $L^{2}$ function with Fourier transform given by:

$$
\hat{f}_{\varepsilon}(\lambda, b)=\hat{F}_{\varepsilon}(\lambda, b) e^{t\left(|\lambda|^{2}+|\delta|^{2}\right) / 2}
$$

We may therefore apply the isometry formula to $F_{\varepsilon}$, giving:

$$
\lim _{R \rightarrow \infty} I\left(R ; F_{\varepsilon}\right)=\left\|f_{\varepsilon}\right\|^{2}=\int_{|\lambda| \leq 1 / \varepsilon}\|\hat{F}(\lambda)\|^{2} e^{t\left(|\lambda|^{2}+|\delta|^{2}\right)} d \mu(\lambda) .
$$


By monotone convergence, we have:

$$
\lim _{\varepsilon \rightarrow 0} \lim _{R \rightarrow \infty} I\left(R ; F_{\varepsilon}\right)=\int_{\mathbb{R}}\|\hat{F}(\lambda)\|^{2} e^{t\left(|\lambda|^{2}+|\delta|^{2}\right)} d \mu(\lambda) .
$$

If the above expression is finite, we have $F=e^{t \Delta / 2} f$, where $f$ is the $L^{2}$ function with the Fourier transform given by:

$$
\hat{f}(\lambda, b)=\hat{F}(\lambda, b) e^{t\left(|\lambda|^{2}+|\delta|^{2}\right) / 2},
$$

thus establishing the claimed surjectivity result.

\section{The Inversion Formula}

In this section, we provide proofs of the general inversion formula (Theorem 4) and the inversion formula for odd-dimensional hyperbolic spaces (Theorem 5).

Proof of Theorem 4. Fix a point $x \in G / K$; let $A_{x}$ be a maximal flat through $x$; and let $\mathfrak{a}_{x}$ be the tangent space at $x$ to $A_{x}$. Since $f^{(x)}$ is invariant under the action of $K_{x}$, we may expand the restriction of $f^{(x)}$ to $A_{x}$ in terms of the spherical functions (relative to the base point $x$ ):

$$
f^{(x)}(a)=\int_{\mathfrak{a}_{x}^{*}} \phi_{\lambda}(a) \widehat{f^{(x)}}(\lambda) \frac{d \lambda}{|c(i \lambda)|^{2}}, \quad a \in A_{x}
$$

(Since, after identifying $\mathfrak{a}_{x}$ with $\mathfrak{a}$, the spherical functions are independent of $x$, we suppress their dependence on $x$ in the notation.) Here, $\widehat{f^{(x)}}$ denotes the spherical Fourier transform of $f^{(x)}$, which is essentially just the Helgason-Fourier transform for $G / K$ restricted to $K$-invariant functions, and $c(\cdot)$ is the Harish-Chandra $c$-function.

Now, the Fourier transform "diagonalizes" the action of the Laplacian $\Delta$ for $G / K$; specifically,

$$
\widehat{\Delta f}(\lambda, b)=\left(|\delta|^{2}+|\lambda|^{2}\right) \hat{f}(\lambda, b)
$$

Thus, the Sobolev space $H^{r}(G / K)$ may be described as the space of functions $f \in L^{2}(G / K)$ for which:

$$
\int_{\mathfrak{a}^{*}}|\hat{f}(\lambda, b)|^{2}\left(|\delta|^{2}+|\lambda|^{2}\right)^{r} d b \frac{d \lambda}{|c(i \lambda)|^{2}}<\infty .
$$

Now, if $f$ is in $H^{r}(G / K)$, then $f^{(x)}$ is also in $H^{r}(G / K)$. (This claim holds because averaging over the action of $K$ commutes with the Laplacian. Alternatively, averaging over the action of $K$ has the effect of averaging $\hat{f}$ over the action of $K$ on $B$, which only reduces the $L^{2}$ norm of $\hat{f}$ over $B$.) In that case, the function:

$$
\left[(I-\Delta)^{r / 2} f^{(x)}\right]^{\wedge}(\lambda)=\left(1+|\delta|^{2}+|\lambda|^{2}\right)^{r / 2} \widehat{f^{(x)}}(\lambda)
$$

belongs to $L^{2}\left(\mathfrak{a},|c(\lambda)|^{-2}\right)$. However, as a consequence of the Gindikin-Karpelevič formula (see, e.g., Section IV.6 of [32]), the function $|c(i \lambda)|^{-2}$ has at most polynomial growth at infinity. Thus, the Cauchy-Schwarz inequality tells us that:

$$
\widehat{f^{(x)}}(\lambda)=\frac{1}{\left(1+|\delta|^{2}+|\lambda|^{2}\right)^{r / 2}} \cdot\left(1+|\delta|^{2}+|\lambda|^{2}\right)^{r / 2} \widehat{f^{(x)}}(\lambda)
$$


is in $L^{1}\left(\mathfrak{a}_{x},|c(i \lambda)|^{-2}\right)$, assuming $r$ is large enough.

Meanwhile, since $F^{(x)}=e^{t \Delta / 2}\left(f^{(x)}\right)$, we have:

$$
F^{(x)}(a)=\int_{\mathfrak{a}} \phi_{\lambda}(a) e^{-t\left(|\lambda|^{2}+|\delta|^{2}\right) / 2} \widehat{f^{(x)}}(\lambda) \frac{d \lambda}{|c(i \lambda)|^{2}}
$$

Thus, by the defining property of the shift operator (see Equations (3.11) and (3.12) in [20]),

$$
D\left(F^{(x)}\right)\left(\exp _{x}(Y)\right)=\int_{\mathfrak{a}} \psi_{\lambda}\left(\exp _{x}(Y)\right) e^{-t\left(|\lambda|^{2}+|\delta|^{2}\right) / 2} \widehat{f^{(x)}}(\lambda) \frac{d \lambda}{|c(i \lambda)|^{2}}
$$

where $\psi_{\lambda}$ is the Euclidean counterpart of the spherical function:

$$
\psi_{\lambda}\left(\exp _{x}(Y)\right)=\frac{1}{|W|} \sum_{w \in W} e^{i\langle\lambda, Y\rangle} .
$$

Here, $W$ is the Weyl group for the symmetric space $G / K$. We normalize the $\psi_{\lambda}$ 's differently from [20] by including a factor of $|W|$ in the denominator in Equation (39). Our shift operator therefore differs by a factor of $|W|$ from that in [20].

Now, by direct calculation,

$$
\int_{\mathfrak{a}} e^{-\langle\lambda, Y\rangle} \frac{e^{-|Y|^{2} /(2 t)}}{(2 \pi t)^{k / 2}} d Y=e^{t|\lambda|^{2} / 2}
$$

Since $\widehat{f^{(x)}}(\lambda)$ is in $L^{1}$, we may expand $\psi_{\lambda}$ as a linear combination of exponentials and apply Fubini's theorem to each of the $|W|$ terms in Equation (38). The result is that:

$$
\begin{aligned}
& e^{t|\delta|^{2} / 2} \int_{\mathfrak{a}_{x}} D\left(F^{(x)}\right)\left(\exp _{x}(i Y)\right) \frac{e^{-|Y|^{2} /(2 t)}}{(2 \pi t)^{k / 2}} d Y \\
& =e^{t|\delta|^{2} / 2} \int_{\mathfrak{a}} e^{t|\lambda|^{2} / 2} e^{-t\left(|\lambda|^{2}+|\delta|^{2}\right) / 2} \widehat{f^{(x)}}(\lambda) \frac{d \lambda}{|c(\lambda)|^{2}} \\
& =f^{(x)}(x) \\
& =f(x),
\end{aligned}
$$

with absolute convergence of the integral.

We now turn to the proof of the inversion formula for $H^{2 n+1}$.

Proof of Theorem 5. Let us now specialize Theorem 4 to the case of an odd-dimensional hyperbolic space, $H^{2 n+1}$. Since the density against that we are integrating in the definition of $J(R, x)$ is $K$-invariant, the result is unchanged if we replace $F$ by $F^{(x)}$. Thus, using polar coordinates and the definition of $\nu_{t}$, we have:

$$
\begin{aligned}
& J(R, x)=c_{n} \int_{0}^{R} F^{(x)}(i r) \nu_{t}(r) \sin ^{2 n} r d r \\
& =c_{n} e^{t n^{2} / 2} \int_{0}^{R} F^{(x)}(i r) \tilde{D}^{*}\left(\frac{e^{-r^{2} /(2 t)}}{\sqrt{2 \pi t}}\right) \sin ^{2 n} r d r .
\end{aligned}
$$


Thus, by Proposition 8,

$$
J(R, x)=\text { B.T. }+e^{t n^{2} / 2} 2 \int_{0}^{R} \tilde{D}\left(F^{(x)}(i r)\right) \frac{e^{-r^{2} /(2 t)}}{(2 \pi t)^{k / 2}} d r .
$$

After converting $2 \int_{0}^{R}$ into $\int_{-R}^{R}$ and applying Theorem 4, we see that the last term on the right-hand side of Equation (41) tends to $f(x)$. It then remains only to show that the boundary terms tend to zero as $R$ tends to infinity in the region $S_{\varepsilon, A}$.

We now turn to the analysis of the boundary terms. We start from the last expression in Equation (40) and use the spherical Fourier transform to express $F^{(x)}(i r)$ as follows:

$$
F^{(x)}(i r)=\int_{\mathbb{R}} \widehat{f^{(x)}}(\lambda) e^{-t\left(|\lambda|^{2}+|\delta|^{2}\right) / 2} \phi_{\lambda}(i r) \frac{d \lambda}{|c(i \lambda)|^{2}}
$$

(compare Equation (37) in the setting of general symmetric spaces of the non-compact type). It is then straightforward to justify reversing the order of integration, provided that we stay away from the poles in the $r$ integral. Thus, we obtain that:

$$
\begin{aligned}
& J(R, x) \\
& =\int_{\mathbb{R}} \widehat{f^{(x)}}(\lambda) e^{-t\left(|\lambda|^{2}+|\delta|^{2}\right) / 2}\left[c_{n} \int_{0}^{R} \phi_{\lambda}(i r) \tilde{D}^{*}\left(e^{t n^{2} / 2} \frac{e^{-r^{2} /(2 t)}}{\sqrt{2 \pi t}}\right) \sin ^{2 n} r d r\right] \frac{d \lambda}{|c(i \lambda)|^{2}} .
\end{aligned}
$$

We now successively integrate by parts in the inner integral in Equation (43), and we must show that the boundary terms can be neglected. Fortunately, the inner integral in Equation (43) is precisely the same as the inner integral in Equation (33), except that $t$ there has been replaced by $t / 2$ here. Thus, by the proof of Theorem 3, each boundary term can be bounded by a constant (independent of $R$ and $\lambda$ ) times $e^{t|\lambda|^{2} / 2}$. Since, by our assumptions on $f$, the function $\widehat{f^{(x)}}(\lambda)$ is in $L^{1}$, we can apply dominated convergence to move the limit as $R$ tends to infinity inside the integral, at which point the boundary terms certainly vanish.

\section{Acknowledgments}

The first author's research was supported in part by NSF grant DMS-1301534. The authors thank the two anonymous referees for a careful reading of the manuscript, which has improved the paper substantially. 


\section{Author Contributions}

Both authors contributed to the research and writing of this paper.

\section{Conflicts of Interest}

The authors declare no conflict of interest.

\section{References}

1. Bargmann, V. On a Hilbert space of analytic functions and an associated integral transform. Comm. Pure Appl. Math. 1961, 14, 187-214.

2. Segal, E.E. Mathematical problems of relativistic physics; Lectures in Applied Mathematics; American Mathematical Society: Providence, RI, USA, 1963.

3. Segal, I.E. The complex wave representation of the free Boson field. In Topics in Functional Analysis: Essays Dedicated to M.G. Krein on the Occasion of His 70th Birthday; Advances in Mathematics Supplementary Studies; Gohberg, I., Kac, M., Eds.; Academic Press: New York, NY, USA, 1978; Volume 3, pp. 321-343.

4. Gross, L. Uniqueness of ground states for Schrödinger operators over loop groups. J. Funct. Anal. 1993, $112,373-441$.

5. Hall, B.C. The Segal-Bargmann "coherent state" transform for compact Lie groups. J. Funct. Anal. 1994, 122, 103-151.

6. Hall, B.C. The inverse Segal-Bargmann transform for compact Lie groups. J. Funct. Anal. 1997, $143,98-116$.

7. Hall, B.C. Harmonic analysis with respect to heat kernel measure. Bull. Amer. Math. Soc. (N.S.) 2001, 38, 43-78.

8. Gross, L.; Malliavin, P. Hall's transform and the Segal-Bargmann map. In Itô's Stochastic Calculus and Probability Theory; Ikeda, N., Watanabe, S., Fukushima, M., Kunita, H., Eds.; Springer: Tokyo, Japan, 1996; pp. 73-116.

9. Hall, B.C.; Sengupta, A.N. The Segal-Bargmann transform for path-groups. J. Funct. Anal. 1998, $152,220-254$.

10. Driver, B.K.; Hall, B.C. Yang-Mills theory and the Segal-Bargmann transform. Comm. Math. Phys. 1999, 201, 249-290.

11. Hall, B.C. Coherent states and the quantization of (1+1)-dimensional Yang-Mills theory. Rev. Math. Phys. 2001, 13, 1281-1305.

12. Wren, K.K. Constrained quantisation and $\theta$-angles. II. Nuclear Phys. B 1998, 521, 471-502.

13. Hall, B.C. Geometric quantization and the generalized Segal-Bargmann transform for Lie groups of compact type. Comm. Math. Phys. 2002, 226, 233-268.

14. Ashtekar, A.; Lewandowski, J.; Marolf, D.; Mourão, J.; Thiemann, T. Coherent state transforms for spaces of connections. J. Funct. Anal. 1996, 135, 519-551.

15. Thiemann, T.; Winkler, O. Gauge field theory coherent states (GCS). II. Peakedness properties. Class. Quantum Gravity 2001, 18, 2561-2636. 
16. Stenzel, M.B. The Segal-Bargmann transform on a symmetric space of compact type. J. Funct. Anal. 1999, 165, 44-58.

17. Akhiezer, D.N.; Gindikin, S.G. On Stein extensions of real symmetric spaces. Math. Ann. 1990, 286, 1-12.

18. Krötz, B.; Stanton, R.J. Holomorphic extensions of representations. I. Automorphic functions. Ann. Math. 2004, 159, 641-724.

19. Krötz, B.; Stanton, R.J. Holomorphic extensions of representations. II. Geometry and harmonic analysis. Geom. Funct. Anal. 2005, 15, 190-245.

20. Krötz, B.; Ólafsson, G.; Stanton, R.J. The image of the heat kernel transform on Riemannian symmetric spaces of the non-compact type. Int. Math. Res. Not. 2005, 1307-1329, doi: 10.1155/IMRN.2005.1307.

21. Faraut, J. Formule de Gutzmer pour la complexification d'un espace riemannien symétrique. Mat. Natur. Rend. Lincei 2002, 13, 233-241.

22. Ólafsson, G.; Schlichtkrull, H. The Segal-Bargmann transform for the heat equation associated with root systems. Adv. Math. 2007, 208, 422-437.

23. Ólafsson, G.; Schlichtkrull, H. Representation theory, Radon transform and the heat equation on a Riemannian symmetric space. In Group Representations, Ergodic Theory, and Mathematical Physics: A Tribute to George W. Mackey; Contemporary Mathematics; American Mathematical Society: Providence, RI, USA, 2008; pp. 315-344.

24. Hall, B.C.; Mitchell, J.J. The Segal-Bargmann transform for non-compact symmetric spaces of the complex type. J. Funct. Anal. 2005, 227, 338-371.

25. Hall, B.C.; Mitchell, J.J. Isometry theorem for the Segal-Bargmann transform on a non-compact symmetric space of the complex type. J. Funct. Anal. 2008, 254, 1575-1600.

26. Hall, B.C.; Mitchell, J.J. The Segal-Bargmann transform for compact quotients of symmetric spaces of the complex type. Taiwanese J. Math. 2012, 16, 13-45.

27. Davies, E.B. Heat Kernels and Spectral Theory; Cambridge University Press: Cambridge, UK, 1989.

28. Leichtnam, E.; Golse, F.; Stenzel, M. Intrinsic microlocal analysis and inversion formulae for the heat equation on compact real-analytic Riemannian manifolds. Ann. Sci. École Norm. Sup. 1996, 29, 669-736.

29. Faraut, J. Analysis on the crown of a Riemannian symmetric space. In Lie Groups and Symmetric Spaces; American Mathematical Society Translations Series 2; American Mathematical Society: Providence, RI, USA, 2003; Volume 210, pp. 99-110.

30. Chalykh, O.; Veselov, A.P. Integrability and Huygens' principle on symmetric spaces. Comm. Math. Phys. 1996, 178, 311-338. 
31. Camporesi, R. Harmonic analysis and propagators on homogeneous spaces. Phys. Rep. 1990, 196, $1-134$.

32. Helgason, S. Groups and Geometric Analysis. Integral Geometry, Invariant Differential Operators, and Spherical Functions. Corrected Reprint of the 1984 Original; Mathematical Surveys and Monographs; American Mathematical Society: Providence, RI, USA, 2000.

(c) 2015 by the authors; licensee MDPI, Basel, Switzerland. This article is an open access article distributed under the terms and conditions of the Creative Commons Attribution license (http://creativecommons.org/licenses/by/4.0/). 